\title{
THE DIFFERENTIAL EFFECTS OF PLASMA FROM TWO GROUPS OF CLINICALLY SIMILAR SCHIZOPHRENIC PATIENTS ON LEARNING BEHAVIOR IN RATS
}

\author{
E. F. Domino, D. F. Caldwell, J. Henke, and R. Henke \\ Department of Pharmacology, University of Michigan, Ann Arbor \\ and Laboratory of Psychobiology, Lafayette Clinic, Detroit, Michigan
}

(Received 19 November 1965)

(Revised 24 February 1966)

\section{INTRODUCTION}

IN THE nine years since HEATH and his associates ${ }^{1}$ reported their findings on taraxein, numerous other laboratories have sought confirmation of the presence of an abnormality unique to the blood of schizophrenic patients. Germane to this research have been attempts to demonstrate behavioral deficits in animals given various blood constituents from schizophrenics. To date, results of such research are equivocal. WINTER and FLATTAKER ${ }^{2}$ and BERGEN et $a l .^{3}$ have obtained evidence of a substance in the blood of schizophrenics which increases response time of rats trained to perform a positively rewarded instrumental response. BISHOP ${ }^{4}$ has reported plasma from schizophrenic patients depresses learning of a conditioned avoidance response in rats. The literature is however, replete with reports failing to substantiate the existence of a factor unique to the blood of schizophrenics capable of affecting behavior. ${ }^{5-8}$

Failure to show evidence of a behavioral impairment in animals following the injection of plasma of schizophrenic patients may reflect the use of inappropriatc test procedures, subject number, subject selection, analysis procedures or any of the other sundry criticisms frequently leveled at negative data. Although general agreement exists among investigators with regard to an adequate use of most of these parameters, considerable variation appears to exist as to what constitutes an appropriate behavioral measure for screening plasma from schizophrenics. In large part, the use of one assessment tcchnique, to the exclusion of another, reflects largely the desire to use a measure proven reliable in one's laboratory. BISHoP ${ }^{9}$ has raised the important question of test validity as it relates to measures of learning used to assay the behavioral effects of plasma. The decision of many investigators to use learning measures appears motivated by laboratory studies at the human level of the effects of schizophrenia upon learning performance. MEDNICK ${ }^{10}$ concluded that schizophrenic patients are superior to normal subjects for tasks of low complexity (e.g. classical conditioning), the reverse being true as task complexity increases. BISHOP ${ }^{9}$ has hypothesized that demonstrations of an agent in the plasma of schizophrenic patients capable of impairing behavior 
might favor use of a 'complex' animal task in preference to simple learning measures. Bishop suggests that for measures of learning, acquisition would represent a more complex phase than the retention or later phase of task performance and thus would be a more valid index of plasma effects. To date, such a distinction appears unwarranted in light of the reports by BERGEN et al., ${ }^{3}$ and BISHOP ${ }^{4}$ which show evidence for a behavioral deficit following the injection of plasma in rats and measured for retcntion and acquisition of a learning task, respectively. It should be further noted that type of reinforcement (viz. positive or negative) appears to be of minor importance in view of the findings of Bergen et al. and Bishop for approach and avoidance learning.

One point concerning task complexity deserves closer scrutiny. Bergen et al. report that plasma from all schizophrenics tested by them depressed performance on their task. These data are in contrast to those of BISHOP ${ }^{9}$ who reported that while grouped data demonstrated learning impairment for rats administered plasma from schizophrenic patients, approximately 33 per cent of the schizophrenic donor's plasma produced faster learning when compared to animals injected with normal plasma. It remains to be answered whether the difference between these two studies is attributable to the degree of purity of the plasma injected (viz. purified plasma fractions in the Bergen et al. study and unfractionated plasma for the Bishop study), the discriminative power of the respective tests or some, as yet, unknown parameter. Such an unknown parameter may be the degree of universality of a blood metabolite in plasma from schizophrenics capable of impairing animal behavior.

Specitically, the hypothesis might be advanced that any blood metabolite in plasma from schizophrenic patients capable of impairing animal behavior may not be universally present in the schizophrenic population. Thus, success or failure to demonstrate a 'psychotoxic' effect in animals administered schizophrenic plasma may resolve itself to a problem based on the selection of the patients being tested. The feasibility of such a hypothesis would be greatly enhanced if independent psychometric or biometric measures could clearly subdivide a population of schizophrenic patients.

The present investigation was designed as a preliminary test of the effect of blood plasma from schizophrenic patients on acquisition of a conditioned avoidance response in rats. Donors for this research were a group of chronic schizophrenic patients from the Lafayette Clinic research ward and housed at Northville State Hospital. Although these patients appear to be clinically homogeneous, they have been found to be differentiable into two discernable subgroups based on several biometric criteria $\left(\right.$ GOTTLIEB $\left.^{11}\right)$. This paper describes the results of research aimed at the question of the importance of such a distinction for studies of 'psychotoxicity' of the plasma from schizophrenics. It is not the intent of this research to validate, by the use of behavioral techniques, the differences found to exist between these subgroups for the various measures listed here; but instead to utilize this subgrouping, based on divergent tests, as possible criteria for testing our hypothesis.

\section{Selection of schizophrenic donors}

\section{MFTHOD}

The criteria for diagnosis of chronic schizophrenia in patients used in this study have been previously described by CRANDALl et al. ${ }^{13}$ All patients tested were from Northville State Hospital and housed in a special research ward under supervision of the Lafayette 
Clinic and received a controlled adcquate diet and daily vitamin supplements. All patients were free of drugs for at least 12 months with the exception of moderate daily amounts of coffee and cigarettes. Duration of hospitalization ranged from a minimum of four years to a maximum of fifteen years.

One group of these patients $(N=6)$ have been found to approach the scores of normal control subjects for the following measures: (a) GSR conditioning, (b) weight discrimination ability, (c) computer averaged photic evoked responses, (d) measures of work output and (e) lactate: pyruvate ratios in chicken erythrocytes following incubation in patients' plasma. Schizophrenics in this group have been labelled subgroup A for convenience in discussion. The second group ( $N=6$ ) from this population performed for these tasks when compared to normals and subgroup A schizophrenics as follows: (a) less GSR conditioning, (b) poorer weight discrimination ability, (c) lower amplitude and shorter latency of photic evoked responses, (d) lower work output, and (e) higher lactate to pyruvate ratios in plasma-incubated chicken erythrocytes. We have labelled these patients subgroup B. Two other tests provided evidence for the division of these schizophrenics into subgroups, although the direction of change differed from the other measures. Subgroup A subjects showed a decreased energy content in the EEG upon frequency analysis with photic stimulation when compared to controls, whereas subgroup B subjects had an increased energy content. Furthermore, subgroup A subjects manifested a reduced amount of electroencephalographic stage 1-REM sleep when compared to either subgroup B or control subjects. Reference made earlier to the clinical similarity between these subgroups, is based on an analysis of seventy-four clinical ratings of course of illness, mental status, symptomatology, and family history conducted on these patients. To date, no clinical variable has been found to clearly and consistently distinguish between these subgroups.

Normal control donors $(N=6)$ in good physical health were selected from volunteers from the laboratory staffs of the Lafayette Clinic.

Blood was drawn from patients and controls in the morning before breakfast, centrifuged at room temperature and refrigerated at $4^{\circ} \mathrm{C}$. within $\frac{1}{2}-1 \mathrm{~h}$ following collection. It remained there until used for either the morning (1-3 h post withdrawal) or afternoon (7-9 h post withdrawal) injections.

\section{Testing procedures}

Seventy male Sprague-Dawley rats weighing between 106 and $200 \mathrm{~g}$ served as subjects. Animals were allowed food and water ad libitum. Six experimental treatments were conducted in the morning and replicated on different subjects in the afternoon to control for time of testing variations in animal behavior. In all, twelve groups of animals were studied. The following six treatments were given: (a) subgroup A schizophrenics' plasma, (b) subgroup B schizophrenics' plasma, (c) normal plasma, (d) isotonic saline with $0.5 \%$ heparin, (e) reconstituted irradiated plasma, and (f) no injection. Plasma from each donor as well as the control solutions were given intraperitoneally. Morning tests were accomplished between the hours of 8 and 10 a.m., afternoon tests between 2 and 4 p.m. Rats were given plasma in an amount equivalent to $10 \mathrm{ml} / \mathrm{kg}$ body weight. Each $S$ remained in the test apparatus for $5 \mathrm{~min}$ following the injection and then was tested for acquisition of the pole 
jump response. A modification of the test procedure of CoOK and WEIDLEY ${ }^{12}$ was used. The pole jump apparatus consisted of a box 9 in. $\times 9$ in. $\times 21$ in. with a grid floor. A pole extended from the top of the box to within three inches of the center of the floor. The pole was of metal $1 \frac{1}{4}$ in. in diameter allowing the $S$ to slide off easily at the termination of a jump response. The grid floor was connected to a $60 \mathrm{c} / \mathrm{s}, 1 \mathrm{~mA}$ shock source supplied through a Foringer grid-shock scrambler. Illumination was provided by a $25 \mathrm{~W}$ bulb. The entire test apparatus was sound damped with a one-way-vision screen. The conditioned stimulus (CS) was a door buzzer of $5 \mathrm{~s}$ duration and was followed by and overlapped with an electric shock unconditioned stimulus (US) of $10 \mathrm{~s}$ duration. A random intertrial interval of $30 \mathrm{~s}$ mean duration was utilized under massed training conditions. A criterion of 9 out of 10 avoidance responses (i.e. pole jump only during the CS interval) was utilized. Testing was terminated if criterion was not achieved in 100 trials, and the number of trials recorded as 100 .

\section{RESULTS}

Data were combined for morning and afternoon test sessions for each of the six experimental treatments when statistical analyses failed to show significant differences for this variable. It was worthy of note however, that subjects tested in the afternoon which received plasma from either normal or schizophrenic patients achieved criterion performance in

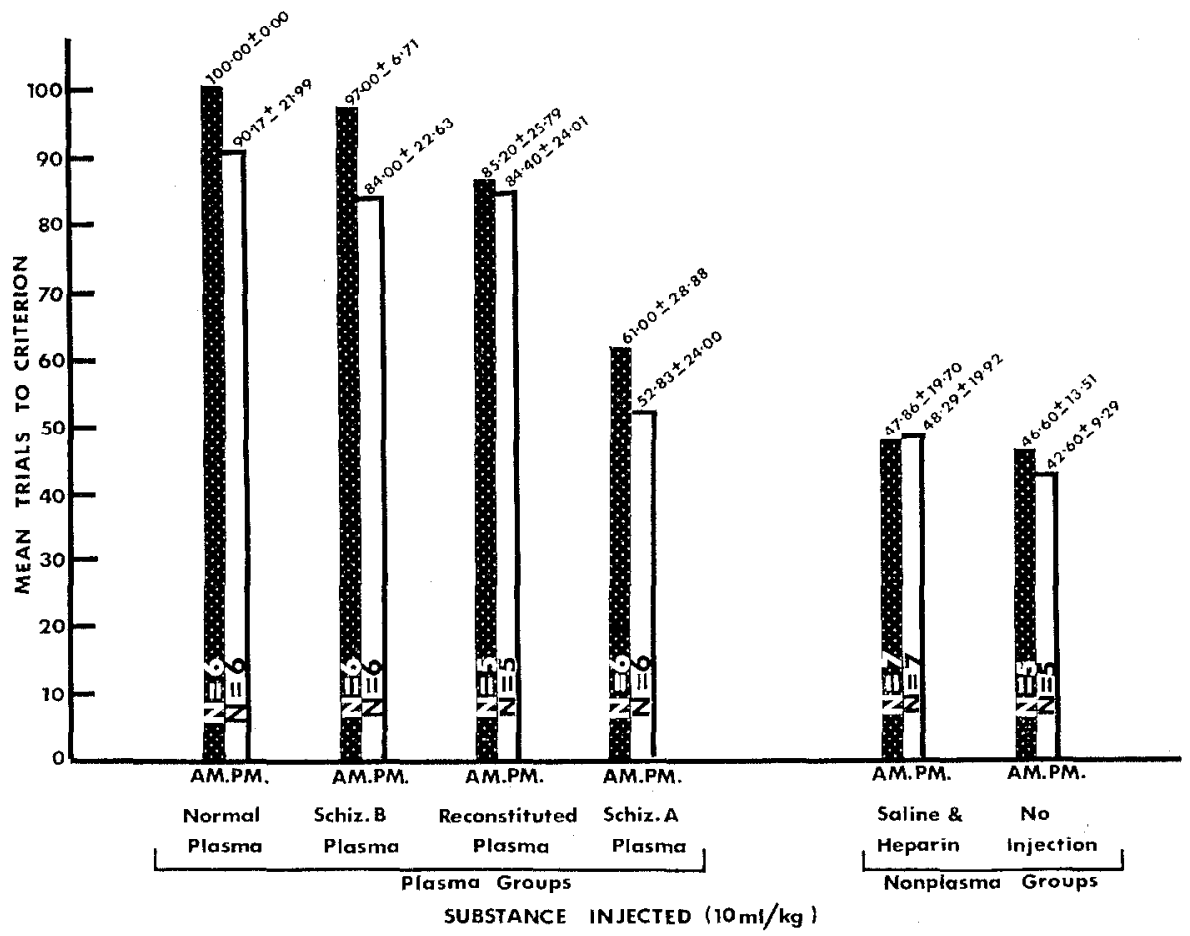

FIG. 1. Mean trials to criterion ( \pm 1 standard deviation) for plasma and nonplasma injected groups on the pole jump test for a.m. and p.m. test sessions. 
approximately ten trials less than the other groups tested in the morning. Figure 1 presents these data for all experimental treatments. Failure to observe any marked performance differences for morning versus afternoon tests for the nonplasma groups indicates the absence of any confounding effect for time of testing (i.e. morning vs. afternoon replication). Furthermore, this observation suggests that time between withdrawal and injection of plasma (i.e. age of plasma post withdrawal) is a variable capable of affecting a behavioral change. Similarly, no significant differences were obtained between groups which received normal, reconstituted, or schizophrenic plasma (i.e. combined data for schizophrenic subgroups A and B), nor were differences observed between the saline-heparin and no injection groups. Data from the saline-heparin and no injection groups were combined to form a nonplasma group $(N=24)$ for purposes of subsequent comparisons. A test of the behavioral effects of plasma revealed that the normal, reconstituted, and combined schizophrenic plasma groups were all significantly slower in attaining performance criterion when com-

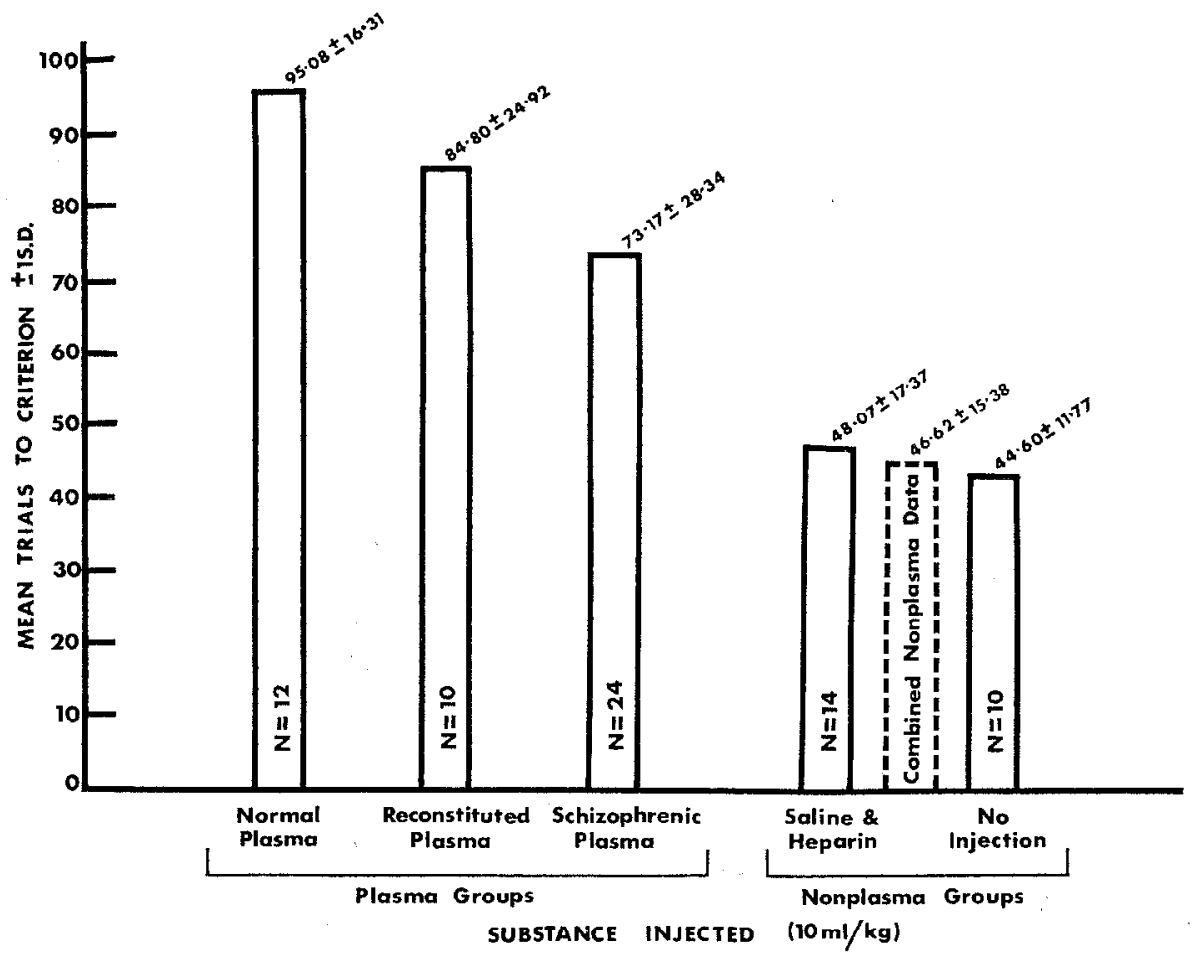

FIG. 2. Comparison of combined a.m. and p.m. data for plasma and nonplasma injections on mean trials to criterion ( \pm 1 standard deviation).

pared to the nonplasma group ( $p<0.002 ; p<0.002$ and $p<0.003$, respectively for the Mann-Whitney $U$ test for nonparametric data). Figure 2 presents performance data for the plasma and combined nonplasma groups. A comparison of all plasma regardless of source $(\bar{X}=81 \cdot 69, N=46)$ to nonplasma $(\bar{X}=46 \cdot 62, N=24)$ was highly significant $(p<0 \cdot 001)$.

Next, the data for schizophrenic subgroups $\mathrm{A}(\bar{X}=56 \cdot 92, N=12)$ and $\mathrm{B}(\bar{X}=90 \cdot 50$, 
$N=12)$ were compared, and each compared separately to the normal $(\bar{X}=95 \cdot 08, N=12)$, reconstituted ( $\bar{X}=84 \cdot 80, N=10)$, and nonplasma treatments $(\bar{X}=46 \cdot 62, N=24)$. Table 1 presents the $p$ values (Mann-Whitney two-tailed $U$-test) computed for these comparisons. It may be seen that animals treated with plasma from schizophrenic subgroup B responded similarly to subjects given normal or reconstituted plasma but, were significantly

TABLE 1. PRobabilities ATtaChEd TO Two GROUP COMPARISONS FOR PLASMA AND NONPLASMA TREATMENTS USING THE MANN-WHITNEY $U$-TEST FOR NON PARAMETRIC DATA

\begin{tabular}{|c|c|c|c|c|}
\hline \multirow{2}{*}{\multicolumn{3}{|c|}{ Substance injected }} & \multicolumn{2}{|c|}{ Schizophrenic plasma } \\
\hline & & & $\begin{array}{l}\text { Subgroup } \mathrm{A} \\
(N=12)\end{array}$ & $\begin{array}{c}\text { Subgroup B } \\
(N=12)\end{array}$ \\
\hline Normal plasma & $(N=12)$ & vs. & $\left\{p<0.002^{*}\right.$ & N.S. \\
\hline Reconstituted plasma & $(N=10)$ & vs. & $\{p<0.05$ & N.S. \\
\hline \multirow[t]{2}{*}{ Non plasma } & $(N=24)$ & vs. & $\{$ N.S. $\dagger$ & $p<0.002$ \\
\hline & Subgroup A & vs. & Subgroup B & $p<0.02$ \\
\hline
\end{tabular}

* Two-tail Mann-Whitney $U$-test.

$\dagger$ Nonsignificant for $p \leq 0 \cdot 10$, two-tail Mann-Whitney $U$-test.

slower learners than animals receiving plasma from subgroup A and the nonplasma controls. These results are in sharp contrast to the performance of animals given plasma from subgroup A who differed significantly from both the normal and reconstituted plasma-treated animals, but not from the nonplasma group.

\section{DISCUSSION}

The results of this investigation clcarly point to a disturbance in ratc of lcarning an avoidance task following the intraperitoneal injection of human plasma. Obviously the site of action for the detrimental effects of human plasma needs to be established. It may well be a local gastrointestinal irritation or any of sundry other causes arising from the injection of a foreign protein or other organic or inorganic substances. The possibility of a direct central action remains, but must be established in future research. It appears to matter little whether such plasma is of long standing or freshly drawn. Furthermore, it would seem, at first glance, that the distinction between normal, reconstituted, or non-subgrouped schizophrenic plasma is not important, for all produced a behavioral effect. However, when, in the present investigation, the schizophrenic group was differentiated into two subgroups based on previous studies, ${ }^{11}$ a striking finding was that plasma from one group lacked apparent signs of impairing learning rate when compared to a saline control group or subjects receiving no injection; whereas, plasma from the other subgroup was virtually indistinguishable in the degree to which it depressed behavior from that of normal or reconstituted plasma. This study is in agreement with the findings of BisHop ${ }^{9}$ for a differential performance in rats given plasma from clinically similar schizophrenics and with GERARD ${ }^{\mathbf{1 4}}$ insomuch as it indicates a likelihood of biologic subgroups of schizophrenics. Perhaps, as 
stated earlier, some of the data in the literature dealing with toxic factors in the blood of schizophrenic patients may be related to this finding.

Whether the failure to observe a learning deficit in rats given the plasma of schizophrenic subgroup A arises from the presence of some blood factor which negates a normally 'psychotoxic' plasma effect or whether their blood lacks some substance which normally affects learning behavior, are only two of numerous possibilities which remain to be investigated. There is little doubt, however, that the learning deficit produced by plasma from subgroup B is not directly related to the biochemical factor responsible for the change in the lactate to pyruvate ratio as outlined by FroHman et al..$^{15,16}$ First, the plasma factor described by Frohman is almost completely destroyed within $3 \mathrm{~h}$ on standing at $4^{\circ} \mathrm{C}$. In the present investigation, the plasma from schizophrenic patients was injected between one and nine hours after withdrawal. Although it was refrigerated most of the time at $4^{\circ} \mathrm{C}$., about $1 \mathrm{~h}$ elapsed prior to the first injections during which it was at or near room temperature. Although some evidence for the loss of a behavioral effect was noted in all three plasma groups by the decrease in trials to criterion for the afternoon versus morning groups, it should be emphasized that this change was observed for all three plasma groups and not exclusively for the schizophrenic subjects. Second, plasma from group B donors, which reportedly contain the factor described by FROHMAN, did not produce a significantly greater difference for mean trials to criterion for the pole jumping test when compared to normal plasma.

One final point deserves mention. It may be noted in this paper for several of the behavioral tests applied to the two schizophrenic subgroups at the Lafayette Clinic and described carlier, ${ }^{11}$ subgroup A patients bchaved similarly to the behavior reported for normal subjects receiving amphetamine-like substances, whereas performance of subgroup B patients was more like that of a group of normals given sedative-like substances. The present data appear to further support this point. Subgroup A plasma injected rats achieved criterion performance in fewer trials than subgroup B subjects. Domino, CALDwELL, and HENKE $^{17}$ have reported increased speed in learning the pole jump response following administration of amphetamine to rats, whereas, decreased learning rates were found to follow the administration of sedatives or psychotomimetics. Although the possible existence of such substances in human plasma is beyond the scope of the present research, the degree of correspondence seem worthy of note.

A note of caution is in order regarding the failure to differentiate the normal, reconstituted and subgroup B treatments. By utilizing the restriction whereby training trials were terminated if the criterion had not been attained in one hundred trials, these groups may have been erroneously made to appear identical. If increased training trials had been administered, these groups may have differed. However, it should be noted from Fig. 1 for the afternoon replication, all groups achieved criterion performance at this time with the differences between the normal, reconstituted, and subgroup B treatment not significant.

\section{SUMMARY}

The effects of plasma from normal subjects and chronic schizophrenic patients were determined on the rate of learning a pole jump response in rats. The animals were trained to 
a buzzer CS and electroshock US. Mean trials to a 90 per cent avoidance criterion were determined using a 100 trial cut off. Plasma and other control solutions were given intraperitoneally in the morning and the procedure repeated in the afternoon with separate groups of rats. Test trials were initiated five minutes after injection. It was found that all plasma, in contrast to saline, produced an increase in the number of trials to learn the avoidance response. No difference in behavioral effects was noted comparing the plasma of all chronic schizophrenics to normals. However, within the chronic schizophrenic population there are subgroups whose plasma was differentially effective in reducing rate of learning. These results are preliminary and subject to continued experimentation. They are presented here to make others aware of the need to consider the existence of possible biologic subgroupings of schizophrenia in future investigations of the plasma-behavior interaction.

\section{REFERENCES}

1. Heath, R. G. Serological Fractions in Schizophrenia. Harper and Row, New York, 1963.

2. WINTER, C. A. and FLATTAKER, L. Effect of blood plasma from psychotic patients upon performance of trained rats. A.M.A. Archs Neurol. Psychiatry 80, 441, 1958.

3. Bergen, J. R. Pennell, R. B., Saravis, C. A. and Hoagland, H. Further experiments with plasma proteins from schizophrenics. Serological Fractions in Schizophrenta (HeATH, R. G., Editor), Harper and Row, New York, 1963.

4. Bishop, M. P. Effects of plasma from schizophrenic subjects upon learning and retention in the rat. Serological Fractions in Schizophrenia (HeAtH, R. G., Editor), Harper and Row, New York, 1963.

5. Ghent, L. and Freedman, A. M. Comparison of effects of normal and schizophrenic serum on motor performance in rats. Am. J. of Psychiat. 115, 465-466, 1958.

6. Stern, J. A., Ulett, G. A. and Smith, Kay. Effect of blood plasma from psychotic patients upon activity levels of white rats. A.M.A. Archs gen. Psychiatry 1, 342-345, 1959.

7. Ferguson, D. C. and Fisher, A. E. Precision timing behavior in cebus apella as a function of intraperitoneal injections of normal, stressed normal and catatonic schizophrenic serum and plasma. Paper presented at the American Psychological Association Meeting, September, 1961.

8. KeTY, S. S. Biochemical Theories of Schizophrenia: Parts I and II. Science 129, 1528-1532; 1590-1596, 1959.

9. Bishor, M. P. Psychotoxic effects of 'schizophrenic plasma in the rat. Doctoral Dissertation, Vanderbilt University, 1961.

10. Mednick, S. A. A learning theory approach to research in Schizophrenia. Psychol. Bull. 55, 316-327, 1958.

11. GotTLIEB, J. S. The biological correlates of the serum protein factor in schizophrenia. Paper presented at the NATO Advanced Study Institute on Molecular Basis of Some Aspects of Mental Activity, Oslo, Norway, Oct. 2-14, 1965.

12. COOK, L. and WEIDLEY, E. F. Behavioral effects of some psychopharmacologic agents. Ann. N.Y. Acad. Sci. 66, 740, 1957.

13. Crandall, R. G., Day, H., Beckett, P. G. S., Chen, C., Brosius, C. D. and Frohman, C. E. Conflicts between treatment and science on a research ward investigating schizophrenia. Can. psychiat. Ass. J. 1965. (In press.)

14. Gerard, R. W. The nosology of schizophrenia: A co-operative study. Behavl. Sci. 9, 311, 1964.

15. Frohman, C. E., Latham, L. K., Beckett, P. G. S. and Gottlieb, J. S. Evidence of a plasma factor in schizophrenia. A.M.A. Archs gen. Psychiatry 2, 255, 1960.

16. Frohman, C. E. CZajkowski, N. P., Luby, E. D., Gottlieb, J. S. and Senf, R. Further evidence of a plasma factor in schizophrenia. A.M.A. Archs. gen. Psychiatry 2, 263, 1960.

17. Domino, E. F., Caluwell, D. F. and Henke, R. Efrects of psychoactive agents on acquisition of conditioned pole jumping in rats. Psychopharmacologia 8, 285-289, 1965. 